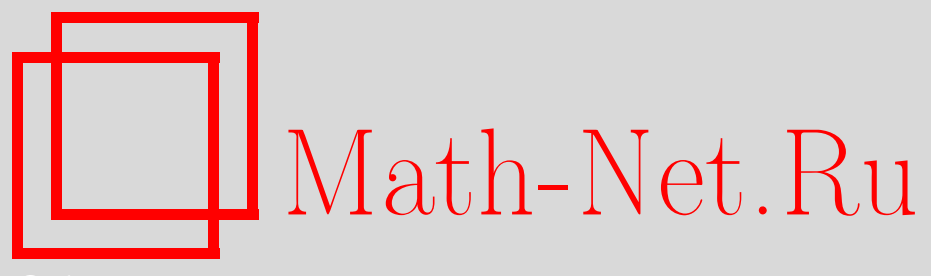

Ю. П. Бибило, Изомонодромные деформации систем линейных дифференциальных уравнений с иррегулярными особенностями, Матем. сб., 2012, том 203, номер 6, 63-80

DOI: https://doi.org/10.4213/sm7861

Использование Общероссийского математического портала Math-Net.Ru подразумевает, что вы прочитали и согласны с пользовательским соглашением http://www . mathnet.ru/rus/agreement

Параметры загрузки:

IP: 3.80 .253 .173

26 апреля 2023 г., $17: 12: 41$

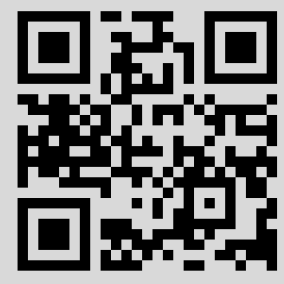




\author{
Ю. П. Бибило
}

\title{
Изомонодромные деформации систем линейных дифференциальных уравнений с иррегулярными особенностями
}

\begin{abstract}
Рассматривается изомонодромная деформация линейных систем дифференциальных уравнений с иррегулярными особенностями. Доказана теорема об общем виде дифференциальной формы, задающей такую деформацию.
\end{abstract}

Библиография: 21 название.

Ключевые слова: изомонодромная деформация, иррегулярные особенности, данные Стокса, форма деформации.

\section{§ 1. Введение}

Вопрос об изучении изомонодромных деформаций систем линейных обыкновенных дифференциальных уравнений впервые был сформулирован Б. Риманом в 1857 г. [1]. В течение длительного периода времени изучались преимущественно изомонодромные деформации линейных систем с фуксовыми особыми точками, в частности Л. Шлезингером, Б. Мальгранжем, А. А. Болибрухом, Б. А. Дубровиным и М. Маццокко и др.

В 1980-х годах М. Джимбо и Т. Мива построили и исследовали деформацию систем с иррегулярными нерезонансными особыми точками [2]. Для описания систем с иррегулярными особыми точками помимо представления монодромии необходим дополнительный набор данных [3], в частности, данные Стокса [4], [5]. Позже изомонодромные деформации систем с иррегулярными особенностями изучались в работах А.Р. Итса и В.Ю. Новокшенова [6], М. В. Федорюка [7], И. М. Кричевера [8] и др.

Исследование изомонодромных деформаций включает в себя следующие проблемы: построение изомонодромных деформаций линейных дифференциальных систем, нахождение общего вида таких деформаций [9], [10], исследование связи изомонодромных деформаций линейных уравнений и систем с нелинейными дифференциальными уравнениями (в частности, нахождение пар Лакса) [6], изомонодромные слияния особых точек [11], [12] и др.

Линейная система с фуксовыми особыми точками (определения приведены ниже) определяется своим представлением монодромии [9], [10], поэтому изомонодромность фуксовой системы естественно понимать как сохранение представления монодромии при деформации. Представление монодромии определяется с точностью до сопряжения на постоянную матрицу (в частности, в зависимости от выбора исходной фундаментальной матрицы). А.А. Болибрух

Работа выполнена в рамках Программы Президента РФ поддержки молодых ученых (грант № MK-4270.2011.1). 
предложил расширить понятие изомонодромной деформации и понимать изомонодромность как сохранение представления монодромии с точностью до сопряжения на постоянную матрицу [10], [13]. Ему удалось получить общий вид дифференциальной 1-формы (в том числе для систем с резонансными фуксовыми особыми точками), задающей изомонодромную деформацию системы с фуксовыми особенностями [10], [14].

В настоящей работе расширенное определение А. А. Болибруха распространяется на случай систем с иррегулярными особыми точками, в результате чего доказана теорема о общем виде дифференциальной формы, задающей изомонодромную деформацию в случае, когда в качестве параметров деформации выбраны положения особых точек.

\section{§ 2. Классификация особых точек}

Рассмотрим линейную систему из $d$ дифференциальных уравнений

$$
\frac{d y}{d z}=A(z) y, \quad y(z) \in \mathbb{C}^{d}
$$

где матрица коэффициентов $A(z)$ мероморфна на $\mathbb{C P}^{1}$ и точки $a_{1}, \ldots, a_{n}-$ ее полюсы.

ОПРЕДЕЛЕНИЕ 1. Особая точка $a_{i}$ называется регулярной особенностью системы (2.1), если внутри каждого сектора конечного раствора с вершиной в точке $a_{i}$ фундаментальная матрица $Y(z)$ системы имеет не более чем степенной рост. В противном случае особая точка $a_{i}$ называется иррегулярной особой точкой системы (2.1).

ОПРедЕЛЕНиЕ 2. Особая точка $a_{i}$ называется фуксовой, если матричная функция $A(z)$ имеет полюс первого порядка в точке $a_{i}$.

Как хорошо известно, фуксова особая точка является регулярной (см., например, [9]).

ОПРЕДЕЛЕНИЕ 3. Целое неотрицательное число $r_{i}$ называется рангом Пуанкаре системы $(2.1)$ в точке $a_{i}$, если разложение в ряд Лорана матрицы $A(z)$ в окрестности $a_{i}$ имеет вид

$$
A(z)=\frac{A_{-r_{i}-1}}{\left(z-a_{i}\right)^{r_{i}+1}}+\cdots+\frac{A_{-1}}{z-a_{i}}+A_{0}+\sum_{k=1}^{\infty} A_{k}\left(z-a_{i}\right)^{k}, \quad A_{-r_{i}-1} \neq 0 .
$$

Преобразование неизвестной функции $y(z)=\Gamma(z) \widetilde{y}(z)$, где $\Gamma(z)$ - мероморфная матричная функция, удовлетворяющая условию $\operatorname{det} \Gamma(z) \not \equiv 0$, называют калибровочным, при этом матрица коэффициентов системы преобразуется следующим образом:

$$
\widetilde{A}(z)=\Gamma^{-1}(z) A(z) \Gamma(z)-\Gamma^{-1}(z) \Gamma^{\prime}(z)
$$

Две системы вида (2.1) с матрицами коэффициентов $A(z)$ и $\widetilde{A}(z)$ называются мероморфно эквивалентными, если найдется некоторое калибровочное преобразование $y(z)=\Gamma(z) \widetilde{y}(z)$ такое, что справедливо соотношение (2.3). 
ОПредЕЛЕНИЕ 4. Ранг Пуанкаре системы (2.1) в точке $a_{i}$ называется минимальным, если он наименьший среди рангов Пуанкаре всех локально мероморфно эквивалентных ей в точке $a_{i}$ систем.

Минимальный ранг Пуанкаре системы (2.1) в регулярной особой точке равен нулю, а в иррегулярной особой точке он строго положителен. Если ранг Пуанкаре регулярной особой точки минимален, то такая точка является фуксовой (см. [9]).

Пусть ранги Пуанкаре системы (2.1) во всех особых точках $a_{1}, \ldots, a_{n}$ минимальны и равны соответственно $r_{1}, \ldots, r_{n}$. И пусть, кроме того, все особые точки отличны от бесконечности. Общий вид системы в таком случае следующий:

$$
\frac{d y}{d z}=A(z) y, \quad A(z)=\sum_{i=1}^{n} \sum_{k=0}^{r_{i}} \frac{A_{-k-1}^{i}}{\left(z-a_{i}\right)^{k+1}}, \quad \sum_{i=1}^{n} A_{-1}^{i}=0 .
$$

Система (2.4) будет основным объектом нашего исследования.

В случае, если все особые точки системы (2.4) фуксовы, система называется фуксовой и представима в виде

$$
\frac{d y}{d z}=\sum_{i=1}^{n} \frac{A_{-1}^{i}}{z-a_{i}} y, \quad \sum_{i=1}^{n} A_{-1}^{i}=0 .
$$

Определим представление монодромии системы (2.4). Выберем произвольную точку $z_{0} \in \mathbb{C P}^{1} \backslash\left\{a_{1}, \ldots, a_{n}\right\}$, и пусть $\gamma$ - некоторая петля, которая начинается и заканчивается в неособой точке $z_{0}$. Аналитически продолжим фундаментальную матрицу $Y(z)$ системы вдоль петли $\gamma$, в результате продолжения получим некоторую, вообще говоря, отличную от $Y(z)$ фундаментальную матрицу $\tilde{Y}(z)$. Так как две фундаментальные матрицы одной и той же линейной системы могут отличаться не более чем на постоянную матрицу, справедливо соотношение $Y(z)=\widetilde{Y}(z) G^{\gamma}$, где $G^{\gamma}$ - невырожденная постоянная матрица. Таким образом, каждой петле $\gamma$ (а точнее, ее гомотопическому классу $[\gamma]$; более подробно см. [10]) соответствует матрица $G^{\gamma}$. Отображение $[\gamma] \rightarrow G^{\gamma}$ задает представление

$$
\chi: \pi_{1}\left(\mathbb{C P}^{1} \backslash\left\{a_{1}, \ldots, a_{n}\right\}, z_{0}\right) \rightarrow \mathbf{G L}(d, \mathbb{C}),
$$

называемое представлением монодромии системы (2.4).

Заметим, что представление $\chi$ зависит от выбора фундаментальной матрицы $Y(z)$. Рассмотрим другую фундаментальную матрицу $Z(z)=Y(z) C$, тогда получим соотношение $Z(z)=\widetilde{Z}(z) C^{-1} G^{\gamma} C$. Это значит, что для системы $(2.4)$ определено не единственное представление монодромии $\chi$, а класс представлений по общему сопряжению на постоянную матрицу.

Пусть $\delta_{i}-$ простая петля, обходящая вокруг только одной особой точки $a_{i}$. Матрица $G^{i}=\left(\chi\left(\delta_{i}\right)\right)^{-1}$ называется матрицей монодромии. Всего $n$ матриц монодромии $G^{1}, \ldots, G^{n}$ порождают группу монодромии с одним групповым равенством $G^{1} \cdots G^{n}=I$, где $I$ - единичная матрица.

Поведение системы в окрестности фуксовой особой точки характеризуют соответствующая матрица монодромии и матрица нормирований. Но в случае иррегулярной особой точки требуется расширенный набор данных. Его описание проводится при помощи локального формального фундаментального решения системы (2.4). 


\section{§ 3. Формальное решение}

ОПРЕДЕЛЕНИЕ 5. Иррегулярная особая точка $a_{i}$ системы (2.4) называется нерезонансной, если все собственные значения матрицы $A_{-r_{i}-1}^{i}$ попарно различны. В противном случае особая точка $a_{i}$ называется резонансной.

ОПРЕДЕЛЕНИЕ 6. Фуксова особая точка $a_{i}$ системы (2.4) называется нерезонансной, если для каждой пары собственных значений матрицы $A_{-1}^{i}$ их разность не является ненулевым целым числом. В противном случае точка $a_{i}$ называется резонансной.

Теорема 1 (см. [15]). Система (2.4) в окрестности иррегулярной особой точки $a_{i} \neq \infty$ имеет формальное фундаментальное решение $\widehat{Y}^{i}(z)$ вида

$$
\widehat{Y}^{i}(z)=\widehat{F}(z)\left(z-a_{i}\right)^{L} e^{Q^{i}(z)} .
$$

При этом, если $a_{i}$ - нерезонансная особая точка, то $\widehat{F}(z)$ - обратимый формальный ряд Тейлора от $z-a_{i}, L$ - диагональная постоянная матрица, $Q^{i}(z)$ - диагональная матрица, элементами которой являются полиномы от $\left(z-a_{i}\right)^{-1}$ с нулевыми свободными членами.

Если $a_{i}$ - резонансная особая точка, то $\widehat{F}(z)$ - обратимый формальный ряд Лорана от $\left(z-a_{i}\right)^{1 / p}$ ( $p$ - натуральное число $)$ с конечной главной частью, $L$ - постоянная матрица, $Q^{i}(z)$ - диагональная матрица, элементами которой являются полиномы от $\left(z-a_{i}\right)^{-1 / p}$ с нулевыми свободными членами.

ОПредЕлЕниЕ 7. Формальное фундаментальное решение (3.1) системы (2.4) называется разветвленным в точке $a_{i}$, если $Q^{i}(z)$ содержит дробные степени $z-a_{i}$. В противном случае - неразветвленным.

Существуют различные способы зафиксировать выбор формального фундаментального решения так, чтобы оно определялось однозначно. В работе [16] доказана следующая теорема о разложении формального фундаментального решения так, что выбор мероморфных инвариантов системы фиксирован и формальный ряд Лорана $\widehat{F}(z)$ не содержит дробных степеней $z$.

ТеОРема 2 (см. [16]). Система (2.4) в окрестности иррегулярной особой точки $a_{i}$ имеет формальное решение вида

$$
\widehat{Y}(z)=\widehat{F}(z)\left(z-a_{i}\right)^{J^{i}} U e^{Q^{i}(z)},
$$

где $\widehat{F}(z)$ и $(\widehat{F}(z))^{-1}$ - формалъные рядъ Лорана от $z-a_{i}$ с конечной главной частью; $Q^{i}(z), U, J^{i}$ - блочные матричь, у которых блочная структура (т.е. размеры соответствующих блоков) совпадает.

Каждый ненулевой блок диагональной матрищъ $Q^{i}(z)$ имеет вид

$$
Q_{j}^{i}(z)=\operatorname{diag}\left(q_{j}^{i}(x) I_{s_{j}}, q_{j}^{i}\left(x \zeta_{j}\right) I_{s_{j}}, \ldots, q_{j}^{i}\left(x \zeta_{j}^{p_{j}-1}\right) I_{s_{j}}\right)
$$

где $q_{j}^{i}-$ полином от $x=\left(z-a_{i}\right)^{-1 / p_{j}}$ с нулевым свободным членом $и \zeta_{j}=$ $e^{2 \pi \mathbf{i} / p_{j}}\left(p_{j}-\right.$ натуральное число, $\mathbf{i}-$ мнимая единица), $I_{s_{j}}-$ единичная матрица размера $s_{j} \times s_{j}$. 
Каждый ненулевой блок блочно-диагональной матрицы $J^{i}$ имеет вид

$$
J_{j}^{i}=\operatorname{diag}\left(J_{s_{j}}^{i}, J_{s_{j}}^{i}+\frac{1}{p_{j}} I_{s_{j}}, \ldots, J_{s_{j}}^{i}+\frac{p_{j}-1}{p_{j}} I_{s_{j}}\right),
$$

где $J_{s_{j}}^{i}$ - матрица в жордановой форме и ее собственнъе значения $\rho_{j}^{1}, \ldots, \rho_{j}^{s_{j}}$ удовлетворяют неравенствам

$$
0 \leqslant \operatorname{Re} \rho_{j}^{m}<\frac{1}{p_{j}}, \quad m=1, \ldots, s_{j} .
$$

Постоянная блочно-диагональная матрица $U=\operatorname{diag}\left(U_{1}, \ldots, U_{N_{Q}}\right)$ содержит ненулевые блоки вида

$$
U_{j}=\left[\zeta_{j}^{(l-1)(k-1)} I_{s_{j}}\right]_{l, k=1}^{p_{j}}, \quad \zeta_{j}=e^{2 \pi \mathbf{i} / p_{j}}
$$

ОпрЕдЕлЕНиЕ 8. Матрица $\widehat{G}^{i}$, определяемая равенством

$$
\widehat{Y}\left(a_{i}+\left(z-a_{i}\right) e^{2 \pi \mathbf{i}}\right)=\widehat{Y}(z) \widehat{G}^{i},
$$

называется матрицей формалъной монодромии системы (2.4) в точке $a_{i}$.

Приведем еще одну форму записи формального фундаментального решения, которая потребуется в дальнейшем. Сделаем замену независимой переменной $\xi=\left(z-a_{i}\right)^{1 / p}$, где $p-$ наименьшее общее кратное чисел $p_{j}$ из формулировки теоремы 2 (натуральное число $p$ будем называть порядком ветвления формального фундаментального решения системы (2.4) в точке $a_{i}$ ). В случае, когда формальное решение неразветвлено, полагаем $\xi=z-a_{i}$. Тогда формальное фундаментальное решение представляется в виде

$$
\widehat{Y}(\xi)=\widehat{\mathscr{F}}(\xi) \xi^{\mathscr{J}^{i}} \exp \mathscr{Q}^{i}(\xi), \quad \mathscr{J}^{i}=\operatorname{diag}\left(\mathscr{J}_{1}^{i}, \ldots, \mathscr{J}_{h}^{i}\right),
$$

где $\mathscr{J}^{i}$ - жорданова матрица, действительные части собственных значений которой лежат в промежутке $[0,1)$, матрица $\mathscr{Q}^{i}(\xi)$ получается из матрицы $Q^{i}(z)$ только заменой независимой переменной, т.е. $\mathscr{Q}^{i}(\xi)=Q^{i}(z)$.

Далее используем построение, повторяющее рассуждения Левеля о построении базиса специального вида (будем называть его левелевским), и приведенное в [17]. Для каждого блока $\mathscr{J}_{k}^{i}$ рассмотрим пространство $\widehat{X}^{k}$, порожденное столбцами матрицы $\widehat{\mathscr{F}}(\xi) \xi^{\mathscr{J}_{k}^{i}}$. Выделим в каждом $\widehat{X}^{k}$ формальный левелевский базис и обозначим через $C_{k}$ матрицу перехода к этому базису. Матрица $C=\operatorname{diag}\left(C_{1}, \ldots, C_{h}\right)$ коммутирует с $\mathscr{Q}^{i}(\xi)$, получаем

$$
\widehat{Y}(\xi) C=\widehat{U}(\xi) \xi^{\Lambda^{i}} \xi^{\widehat{E}^{i}} \exp \mathscr{Q}^{i}(\xi),
$$

где $\Lambda^{i}$ - блочная диагональная матрица с целочисленными элементами, блочная структура которой согласована с блочной структурой $\mathscr{J}^{i}$, и внутри каждого блока диагональные элементы упорядочены по невозрастанию. Новая матрица $\widehat{E}^{i}$ коммутирует с $Q^{i}(z)$, кроме того, матрица $\xi^{\Lambda^{i}} \widehat{E}^{i} \xi^{-\Lambda^{i}}$ голоморфна при $\xi=0$ (см. [17]).

Элементы матрицы $\Lambda^{i} / p$ называются формальными нормированиями системы (2.4) в точке $a_{i}$.

ОПРЕДЕЛЕНиЕ 9. Если в разложении (3.8) формальный ряд $\widehat{U}(\xi)=U_{o}+$ $U_{1} \xi+\cdots$ таков, что $\operatorname{det} U_{0} \neq 0$, то особую точку $a_{i}$ будем называть формально фуксовой иррегулярной особой точкой системы (2.4). 


\section{§ 4. Данные Стокса}

Формальные инварианты (относительно формальных мероморфных преобразований) $J^{i}, Q^{i}(z)$ недостаточно полно описывают систему (2.4) в окрестности иррегулярной особой точки $a_{i}$. Для более точного описания поведения системы в окрестности особой точки вводится дополнительный набор данных, называемых данными Стокса.

Формальное решение, вообще говоря, является расходящимся рядом. В [15] приводится теорема о том, что формальное решение является асимптотическим разложением некоторого фундаментального решения в каждом достаточно узком секторе с вершиной в особой точке.

Рассмотрим иррегулярную особую точку $a_{i}$. Пусть $\widehat{Y}(z)$ - некоторое формальное фундаментальное решение системы $(2.4)$ в окрестности точки $a_{i}$. Любое формальное фундаментальное решение представимо в виде

$$
\widehat{Y}(z)=\widehat{F}(z)\left(z-a_{i}\right)^{J^{i}} U e^{Q^{i}(z)} C_{0},
$$

где $\widehat{F}(z)$ - формальный ряд Лорана, $C_{0}$ - постоянная невырожденная матрица, $Q^{i}(z)=\operatorname{diag}\left(q_{1}^{i}(z) I_{s_{1}}, \ldots, q_{l}^{i}(z) I_{s_{l}}\right)$ и все функции $q_{j}^{i}(z)$ различны (см. [4]).

Определим секторы и матрицы Стокса аналогично [4] (данные Стокса могут быть определены более общим способом, приведенным в [5]). Рассмотрим разность двух неравных диагональных элементов матрицы $Q^{i}(z)$

$$
q_{j}^{i}(z)-q_{h}^{i}(z)=b \frac{1}{\left(z-a_{i}\right)^{m}}+\cdots, \quad b \neq 0,
$$

где $m$ - некоторое рациональное положительное число.

Обозначим главное значение $\arg b$ через $\beta$. Тогда при $z \rightarrow a_{i}$ вдоль направления $\tau=\arg \left(z-a_{i}\right)$ имеем

$$
\left|z-a_{i}\right|^{m} \operatorname{Re}\left(q_{j}^{i}(z)-q_{h}^{i}(z)\right) \rightarrow|b| \cos (\beta-m \tau)=c .
$$

Если значение $c$ равно нулю, то направление $\tau$ называется направлением Сток$c a$. Заметим, что $c=0$ при $\beta-m \tau \equiv-\pi / 2 \bmod \pi$, следовательно, направления Стокса определяются совокупностью равенств

$$
\tau \equiv \frac{\beta+\pi / 2}{m} \quad \bmod \frac{2 \pi}{m}, \quad \tau \equiv \frac{\beta-\pi / 2}{m} \bmod \frac{2 \pi}{m} .
$$

Выберем покрытие проколотой окрестности $\dot{D}\left(a_{i}\right)$ секторами

$$
\Omega_{1}\left(a_{i}\right), \ldots, \Omega_{N_{i}}\left(a_{i}\right), \quad \Omega_{k}\left(a_{i}\right)=\left\{z: \varphi_{k}^{-}<\arg \left(z-a_{i}\right)<\varphi_{k}^{+}\right\} \cap \dot{D}\left(a_{i}\right)
$$

(будем их называть секторами Стокса) такими, что в каждом секторе $\Omega_{k}\left(a_{i}\right)$ содержится только одно направление Стокса (т.е. найдется $z \in \Omega_{k}\left(a_{i}\right)$ такое, что $\tau=\arg \left(z-a_{i}\right)$ удовлетворяет одному из равенств $\left.(4.1)\right), \varphi_{k}^{-}, \varphi_{k}^{+}-$соседние с ним направления Стокса и в пересечениях секторов $\Omega_{k}\left(a_{i}\right) \cap \Omega_{k^{\prime}}\left(a_{i}\right), k \neq k^{\prime}$, направлений Стокса не содержится.

Секторы Стокса, как показывает следующая теорема, важны для понимания связи формального фундаментального решения и "настоящего". 
Tеорема 3 (см. [15]). Формалъное фундаменталъное решение $\widehat{Y}(z)$ системъ (2.4) в каждом секторе Стокса $\Omega_{k}\left(a_{i}\right)$ является асимптотическим разложением фундаментального решения $Y_{k}^{i}(z)$ системы (2.4).

В формулировке теоремы слова о том, что формальное фундаментальное решение $\widehat{Y}(z)$ является асимптотическим разложением фундаментального решения $Y_{k}^{i}(z)$ в секторе $\Omega_{k}\left(a_{i}\right)$, понимаются следующим образом. Фундаментальное решение $Y_{k}^{i}(z)$ таково, что при $z \in \Omega_{k}\left(a_{i}\right)$ для любого $V \in \mathbb{Z}, V \geqslant-v$, справедливо

$$
\left\|Y_{k}^{i}(z)\left(\left(z-a_{i}\right)^{J^{i}} U \exp Q^{i}(z) C_{0}\right)^{-1}-\sum_{\nu=-v}^{V} F_{\nu}\left(z-a_{i}\right)^{\nu}\right\| \leqslant \text { const } \cdot\left|z-a_{i}\right|^{V+1},
$$

где

$$
\widehat{F}(z)=\sum_{\nu=-v}^{\infty} F_{\nu}\left(z-a_{i}\right)^{\nu}
$$

Фундаментальное решение $Y_{k}^{i}(z)$ из теоремы 3 будем называть каноническим в секторе $\Omega_{k}\left(a_{i}\right)$.

Рассмотрим пересечение секторов $\Omega_{k}\left(a_{i}\right) \cap \Omega_{k+1}\left(a_{i}\right)$, тогда соответствующие канонические фундаментальные решения связаны соотношением

$$
Y_{k+1}^{i}(z)=Y_{k}^{i}(z) S_{k}^{i}, \quad z \in \Omega_{k}\left(a_{i}\right) \cap \Omega_{k+1}\left(a_{i}\right),
$$

как фундаментальные решения одной и той же линейной системы. Постоянная матрица $S_{k}^{i}$ называется матрищей Cтокса. В определении матрицы $S_{N_{i}}^{i}$ полагаем $Y_{N_{i}+1}^{i}(z)=Y_{1}^{i}(z) \widehat{G}^{i}$. Наложив некоторые дополнительные нормализующие условия на матрицы $C_{0} S_{k}^{i} C_{0}^{-1}$, можно добиться, чтобы набор фундаментальных решений $Y_{1}^{i}, \ldots, Y_{N_{i}}^{i}$, удовлетворяющий (4.3), был единственным и зависел только от выбора исходного формального решения $\widehat{Y}(z)$ (см. [4]). Фундаментальное решение $Y_{k}^{i}(z)$ будем называть каноническим в секторе $\Omega_{k}\left(a_{i}\right)$.

Заметим, что фундаментальное решение $Y(z)$, удовлетворяющее соотношению

$$
Y(z)=Y_{1}^{i}(z), \quad z \in \Omega_{1}\left(a_{i}\right)
$$

связано с каноническими решениями следующим образом:

$$
\begin{aligned}
& Y(z)=Y_{1}^{i}(z), \quad z \in \Omega_{1}\left(a_{i}\right) \\
& Y(z)=Y_{2}^{i}(z)\left(S_{1}^{i}\right)^{-1}, \quad z \in \Omega_{2}\left(a_{i}\right) \\
& \ldots \ldots \ldots \ldots \ldots \ldots \ldots \ldots \ldots \ldots \ldots \ldots \cdots \cdots \\
& Y(z)=Y_{N_{i}}^{i}(z)\left(S_{1}^{i} \cdots S_{N_{i}-1}^{i}\right)^{-1}, \quad z \in \Omega_{N_{i}}\left(a_{i}\right) .
\end{aligned}
$$

Продолжим аналитически фундаментальное решение $Y(z)$, удовлетворяющее равенству (4.4), около особой точки $a_{i}$. Тогда получим следующее соотношение, связывающее матрицы монодромии и формальной монодромии,

$$
\widehat{G}^{i}\left(S_{1}^{i} S_{2}^{i} \cdots S_{N_{i}}^{i}\right)^{-1}=G^{i} .
$$


ЗАмечАниЕ 1. Часто в качестве набора канонических решений выбирают набор фундаментальных решений в виде (3.2), т.е.

$$
{ }^{s t} Y_{k}^{i}(z)=F_{k}(z)\left(z-a_{i}\right)^{J^{i}} U e^{Q^{i}(z)}, \quad z \in \Omega_{k}\left(a_{i}\right) .
$$

Будем называть такой набор ${ }^{s t} Y_{1}^{i}, \ldots,{ }^{s t} Y_{N_{i}}^{i}$ стандартным набором канонических решений. Тогда фундаментальное решение $Y(z)$, относительно которого определено представление монодромии, и каноническое решение ${ }^{s t} Y_{1}^{i}(z)$, связаны равенством $Y(z)={ }^{s t} Y_{1}^{i}(z) C^{i}, z \in \Omega_{1}\left(a_{i}\right)$. Постоянная невырожденная матрица $C^{i}$, определяемая этим равенством, называется матрицей связи. Матрицы Стокса, определенные выше, и матрицы Стокса, определенные при помощи стандартного набора канонических решений, равны с точностью до сопряжения на соответствующую матрицу связи.

\section{§5. Допустимые деформации}

Рассмотрим семейство систем линейных дифференциальных уравнений (предполагаем, что оно существует), которое голоморфно зависит от $t \in D\left(t^{0}\right)$, где $D\left(t^{0}\right)$ - окрестность точки $t^{0}$ в пространстве параметров,

$$
\frac{d y}{d z}=A(z, t) y, \quad A(z, t)=\sum_{j=1}^{n} \sum_{k=1}^{r_{j}+1} \frac{A_{-k}^{j}(t)}{\left(z-a_{j}(t)\right)^{k}}, \quad \sum_{j=1}^{n} A_{-1}^{j}(t)=0 .
$$

Кроме того, пусть выполнено равенство $A\left(z, t^{0}\right)=A(z)$, где $A(z)$ - матрица коэффициентов системы (2.4).

Сделаем несколько предположений относительно семейства систем (5.1).

1) $a_{1}(t), \ldots, a_{n}(t)$ - голоморфные функции от $t$ такие, что $a_{i}(t) \neq a_{j}(t)$ для любого $t \in D\left(t^{0}\right)$ (другими словами, количество особых точек не меняется).

2) Ранги Пуанкаре $r_{1}, \ldots, r_{n}$ системы (5.1) в особых точках $a_{1}(t), \ldots, a_{n}(t)$ не изменяются и остаются равными минимальным рангам Пуанкаре.

3) При всех значениях параметра $t \in D\left(t^{0}\right)$ в окрестности каждой особой точки соответствующей системы (5.1) можно выбрать секторы Стокса таким образом, что секторы Стокса, соответствующие разным значениям параметра $t$, переходят друг в друга параллельным переносом.

ОПРЕДЕЛЕНИЕ 10. Семейство систем (5.1) с учетом предположений 1)-3) будем называть допустимой деформацией системы (2.4).

В дальнейшем нам потребуется следующая теорема.

Теорема 4. Пусть $Y(z, t)$ - голоморфное по $(z, t)$ фундаментальное решение допустимой деформации (5.1), и формальное фундаментальное решение $\widehat{Y}(z, t)$ является его асимптотическим разложением в секторе $\Omega_{1}\left(a_{i}\right)$. Тогда:

1) асимптотическое разложение равномерно в $D\left(t^{0}\right)$;

2) формальное фундаментальное решение $\widehat{Y}(z, t)$ голоморфно по $t$.

Условие 2) понимается в следующем смысле. Коэффициенты формального ряда, входящего в $\widehat{Y}(z, t)$, голоморфны по $t$, и остальные множители, входящие в $\widehat{Y}(z, t)$, также голоморфны по $t$. 
Доказательство теоремы 4 опирается на теорему о существовании формального фундаментального решения аналитичного по набору параметров. Первоначально следующее утверждение было получено Д. Баббитом и В. Варадаражаном и позднее несколько расширено Р. Шафке [18].

Теорема 5. Пусть при каждом значении $t$ система (5.1) имеет формальное фундаментальное решение, показатель экспоненты которого равен матрице $Q^{i}(z, t)=\operatorname{diag}\left(q_{1}^{i}(z, t), \ldots, q_{p}^{i}(z, t)\right)$, и для матриць $Q^{i}(z, t)$ выполненъь условия:

1) она аналитична по z и по $t$;

2) для всех $k, j$ рациональная степень $\operatorname{deg}\left(q_{k}^{i}(z, t)-q_{j}^{i}(z, t)\right)$ не зависит от $t$.

Тогда найдется окрестность $D\left(t^{0}\right)$ в пространстве параметров и формальное фундаментальное решение вида

$$
\widehat{Y}(z, t)=\widehat{F}(z, t)\left(z-a_{i}\right)^{J^{i}(t)} U e^{Q^{i}(z, t)}
$$

такое, что матричная функиия $J^{i}(t)$ и формальный ряд $\widehat{F}(z, t)$ голоморфно зависят от $t$.

Заметим, что условие 2) теоремы 5 выполнено для допустимой деформации (5.1). Действительно, если условие 2) нарушается, количество направлений Стокса изменится, и следовательно, изменится количество секторов Стокса.

Приведем еще две общие теоремы о равномерных асимптотических разложениях из [15].

ОПРЕДЕЛЕНИЕ 11. Функция $f(z, t)$ имеет равномерное по $t \in D\left(t^{0}\right)$ асимптотическое разложение $\sum_{k=0}^{+\infty} x_{k}(t)\left(z-a_{i}\right)^{k}$ в секторе $\Omega=\left\{z: \alpha \leqslant \arg \left(z-a_{i}\right) \leqslant \beta\right.$, $\left.0<\left|z-a_{i}\right| \leqslant \rho\right\}$, если для всех целых $V>0$ существует предел

$$
\lim _{z \rightarrow a_{i}, z \in \Omega}\left(z-a_{i}\right)^{-V}\left(f(z, t)-\sum_{k=0}^{V} x_{k}(t)\left(z-a_{i}\right)^{k}\right)=0,
$$

равномерный относительно $t \in D\left(t^{0}\right)$.

ТЕОрема 6. Пусть функиия $f(z, t)$ голоморфна по $(z, t)$ в $\Omega \times D\left(t^{0}\right)$, где $\Omega=\left\{z: \alpha \leqslant \arg \left(z-a_{i}\right) \leqslant \beta, 0<\left|z-a_{i}\right| \leqslant \rho\right\}$, и у нее есть равномерное асимптотическое разложение

$$
f(z, t) \sim \sum_{k=0}^{\infty} x_{k}(t)\left(z-a_{i}\right)^{k}, \quad z \rightarrow a_{i}, \quad z \in \Omega .
$$

Тогда все коэфбиииенты $x_{k}(t)$ голоморфны в $D\left(t^{0}\right)$. Кроме того,

$$
\frac{\partial f(z, t)}{\partial t_{j}} \sim \sum_{k=0}^{\infty} \frac{\partial x_{k}(t)}{\partial t_{j}}\left(z-a_{i}\right)^{k}
$$

равномерно на любом собственном компактном подмножестве $\mathscr{K}$ множества $D\left(t^{0}\right)$. 
ТеОРема 7. Пусть $x_{k}(t), k=0,1, \ldots$, голоморфны в $D\left(t^{0}\right)$ u $\Omega$ - произвольный сектор, $\Omega=\left\{z: \alpha \leqslant \arg \left(z-a_{i}\right) \leqslant \beta, 0<\left|z-a_{i}\right|<\rho\right\}$. Тогда найдется функиия $f(z, t)$, голоморфная по обеим переменным в $\Omega \times D\left(t^{0}\right)$ и такая, что для нее имеется равномерное в $D\left(t^{0}\right)$ асимптотическое разложение

$$
f(z, t) \sim \sum_{k=0}^{\infty} x_{k}(t)\left(z-a_{i}\right)^{k}, \quad z \rightarrow a_{i}, \quad z \in \Omega .
$$

ДокаЗАТЕЛЬСтво тЕоремы 4. По теореме 5 система (5.1) имеет аналитичное формальное фундаментальное решение $\widehat{X}(z, t)$. Формальное фундаментальное решение $\widehat{X}(z, t)$, в свою очередь, является равномерным в $D\left(t^{0}\right)$ асимптотическим разложением некоторого фундаментального решения $X(z, t)$, $z \in \Omega_{1}\left(a_{i}\right)$. Следовательно, фундаментальные решения $X(z, t)$ и $Y(z, t)$ связаны соотношением $Y(z, t)=X(z, t) C_{0}(t)$, где $C_{0}(t)$ - невырожденная матрица. И значит, фундаментальное решение имеет равномерное асимптотическое разложение $\widehat{Y}(z, t)=\widehat{X}(z, t) C_{0}(t), z \in \Omega_{1}\left(a_{i}\right)$, коэффициенты формального ряда $\widehat{F}(z, t)$ которого голоморфны по $t \in D\left(t^{0}\right)$.

\section{§ 6. Изомонодромные деформации}

ОпРЕДЕЛЕНиЕ 12. Допустимая деформация (5.1) системы (2.4) называется изомонодромной, если для каждого параметра $t \in D\left(t^{0}\right)$ найдется фундаментальное решение $Y(z, t)$ системы (5.1) такое, что выполнены следующие условия:

1) представление монодромии, определенное при помощи $Y(z, t)$, совпадает с представлением монодромии $\chi$ системы (2.4);

2) в окрестности каждой иррегулярной особой точки $a_{i}(t)$ найдутся набор канонических фундаментальных решений системы $Y_{1}^{i}(z, t), \ldots, Y_{N_{i}}^{i}(z, t)$ такой, что $Y_{1}^{i}(z, t)=Y(z, t)$ для $z \in \Omega_{1}\left(a_{i}\right)$, и определяющий набор матриц Стокса $S_{1}^{i}, \ldots, S_{N_{i}}^{i}$, совпадающий с набором матриц Стокса системы (2.4).

Заметим, что определение 12 согласовано с определением изомонодромных деформаций системы общего положения (т.е. системы, у которой все особенности фуксовы либо нерезонансные иррегулярные). Обычно в таком определении выбор фундаментальной матрицы фиксирован $Y(\infty, t)=I$ и в качестве канонических решений выбираются стандартные канонические решения. Тогда деформация называется изомонодромной, если система остается системой общего положения при деформации и ее матрицы монодромии, матрицы Стокса и матрицы связи постоянны.

Положим $t=\left(a_{1}, \ldots, a_{n}\right)$, т.е. в дальнейшем параметрами деформации будут служить положения особых точек.

Теорема 8. Пусть $t=\left(a_{1}, \ldots, a_{n}\right)$ u $(5.1)$ - изомонодромная деформация. Тогда найдется фундаментальное решение $Y(z, t)$ семейства (5.1) голоморфное по $t \in D\left(t^{0}\right)$ такое, что для него выполнены условия 1), 2) из определения 12 .

ДокАЗАТЕЛьство. Допустим, деформация (5.1) изомонодромна. Следовательно, найдется изомонодромная матрица $Y(z, t)$, и для каждой точки $a_{i}$ выполнено $Y_{1}^{i}(z, t)=Y(z, t), z \in \Omega_{1}\left(a_{i}\right)$. 
Согласно теореме Коши существует фундаментальная матрица $\widetilde{Y}(z, t)$ голоморфная по совокупности переменных $z$ и $t$. Для любого фиксированного значения параметра $t$ выполнено соотношение $\widetilde{Y}(z, t)=Y(z, t) C(t)$. Тогда матрицы монодромии и матрицы Стокса, определенные при помощи $\widetilde{Y}(z, t)$, имеют вид

$$
G^{i}(t)=C^{-1}(t) G^{i} C(t), \quad S_{k}^{i}(t)=C^{-1}(t) S_{k}^{i} C(t) .
$$

Обозначим группу $H=\left\{A \in G L(p, C): A G^{i} A^{-1}=G^{i}, A S_{k}^{i} A^{-1}=S_{k}^{i}\right\}$. Далее, аналогично случаю деформаций фуксовых систем (см. доказательство теоремы 13.1 из [9]) можно построить голоморфную по $t$ матрицу $\widetilde{C}(t)$ такую, что $C(t) \widetilde{C}(t) \in H$ и ${ }^{\text {new }} Y(z, t)=\widetilde{Y}(z, t) \widetilde{C}(t)$ - голоморфное изомонодромное фундаментальное решение, поскольку матрица $\widetilde{C}(t)$ такова, что выполнены равенства

$$
\widetilde{C}^{-1}(t) G^{i}(t) \widetilde{C}(t)=G^{i}, \quad \widetilde{C}^{-1}(t) S_{k}^{i}(t) \widetilde{C}(t)=S_{k}^{i}
$$

Зададим новую систему канонических решений

$$
{ }^{\text {new }} Y_{k}^{i}(z, t)=\widetilde{Y}_{k}^{i}(z, t) \widetilde{C}(t)=Y_{k}^{i}(z, t) C(t) \widetilde{C}(t),
$$

она определяет набор матриц Стокса $S_{k}^{i}$ по построению. Каждое каноническое решение голоморфно зависит от $t$, так как

$$
\begin{aligned}
{ }^{\text {new }} Y_{k}^{i}(z, t) & =Y_{k}^{i}(z, t) C(t) \widetilde{C}(t)=Y(z, t) S_{1}^{i} \cdots S_{k-1}^{i} C(t) \widetilde{C}(t) \\
& =\widetilde{Y}(z, t) S_{1}^{i}(t) \cdots S_{k-1}^{i}(t) \widetilde{C}(t)=\widetilde{Y}(z, t) \widetilde{C}(t) S_{1}^{i} \cdots S_{k-1}^{i},
\end{aligned}
$$

а произведение $\widetilde{Y}(z, t) \widetilde{C}(t) S_{1}^{i} \cdots S_{k-1}^{i}$ голоморфно по $t$.

ЗАмечАниЕ 2. В работе [14] для деформации, особые точки которой неразветвлены, построена изомонодромная матрица в случае, когда параметрами деформации служат $t=\left(\beta_{1}, \ldots, \beta_{H}\right)$, где $\beta_{1}, \ldots, \beta_{H}$ - коэффициенты полиномов от $\left(z-a_{i}\right)^{-1}$, являющихся элементами матриц $Q^{i}(z)$, за исключением коэффициентов при старших степенях.

В настоящей работе задача построения изомонодромной деформации системы с иррегулярными особыми точками не решается. Изомонодромная деформация системы общего положения впервые была построена М. Джимбо и Т. Мивой [2].

Построение изомонодромной деформации системы с иррегулярными резонансными особыми точками, ранги Пуанкаре которых минимальны, более трудно. Мальгранж расширил результат М. Джимбо и Т. Мивы и доказал существование изомонодромной деформации для системы с иррегулярными особыми точками такими, что каждому собственному значению матрицы $A_{-r_{i}-1}^{i}$ соответствует только один жорданов блок [19]. Основываясь на результатах Б. Мальгранжа, В. Ю доказала существование изомонодромной деформации произвольной иррегулярной системы размера $2 \times 2$ (см. [20]). В работах М. Бертолы, М. Мо [21] и М. В. Федорюка [7] строятся изомонодромные деформации системы с иррегулярными особыми точками при специальных условиях на матрицу коэффициентов. 


\section{§ 7. Локальный вид формы деформации}

Справедлива следующая теорема, доказательство которой приведено в 99 .

Теорема 9. Допустимая деформачия (5.1), $t=\left(a_{1}, \ldots, a_{n}\right)$, является изомонодромной тогда и только тогда, когда существует матричная дифференциальная мероморфная 1-форма $\omega$, определенная на $\mathbb{C P}^{1} \times D\left(t^{0}\right)$, с особенностями вдоль гиперплоскостей $\left\{z-a_{i}=0\right\}$ такая, что:

1) $\omega=A(z, t) d z$, при каждом фиксированном значении параметра $t \in D\left(t^{0}\right)$;

2) $d \omega=\omega \wedge \omega$.

Дифференциальная матричная 1-форма $\omega$ из теоремы 9 называется формой, задающей деформацию системы (2.4) (кратко - формой деформации). В силу теоремы 9 возникает вопрос, каков общий вид такой формы деформации.

Пусть $Y(z, t)$ - изомонодромная фундаментальная матрица, голоморфная по $z$ и по $t$. Зададим дифференциальную 1 -форму $\omega=d Y(z, t) Y^{-1}(z, t)$. Она однозначна, так как матрицы монодромии, определенные при помощи фундаментального решения $Y(z, t)$, постоянны. Дифференциальная 1-форма $\omega$ имеет асимптотическое разложение по степеням $z-a_{i}$ одинаковое во всех секторах Стокса $\Omega_{k}\left(a_{i}\right)$. Действительно, в секторах Стокса выполнены равенства (4.5). Так как матрицы Стокса постоянны по $z$ и по $t$, получаем

$$
d Y(z, t) Y^{-1}(z, t)=d Y_{k}^{i}(z, t)\left(Y_{k}^{i}(z, t)\right)^{-1}, \quad z \in \Omega_{k}\left(a_{i}\right) .
$$

Кроме того, по теореме 6 получаем $d Y_{k}^{i}(z, t) \sim d \widehat{Y}(z, t), z \in \Omega_{k}\left(a_{i}\right)$. Таким образом, дифференциальная форма $\omega$ имеет следующее асимптотическое разложение:

$$
\omega=d Y(z, t) Y^{-1}(z, t) \sim d \widehat{Y}(z, t) \widehat{Y}^{-1}(z, t), \quad z \rightarrow a_{i} .
$$

Исследуем структуру дифференциальной 1-формы $\omega$ в окрестности гиперплоскости $\left\{z-a_{i}=0\right\}$ :

$$
\begin{gathered}
\omega=\omega_{1}+\omega_{2}, \quad \omega_{1}=\frac{\partial Y(z, t)}{\partial\left(z-a_{i}\right)} Y^{-1}(z, t) d\left(z-a_{i}\right), \\
\omega_{2}=\sum_{j=1}^{n} \frac{\partial Y(z, t)}{\partial a_{j}} Y^{-1}(z, t) d a_{j} .
\end{gathered}
$$

Из (7.1) сразу следует равенство

$$
\omega_{1}=\sum_{k=1}^{r_{i}+1} \frac{A_{-k}^{i}(t)}{\left(z-a_{i}\right)^{k}} d\left(z-a_{i}\right)+\psi_{i}(z, t) d\left(z-a_{i}\right),
$$

где $\psi_{i}(z, t)$ - некоторая голоморфная по $z$ и $t$ матрица.

Дифференциальная 1-форма $\omega_{2}$ имеет асимптотическое разложение

$$
\sum_{j=1}^{n} \frac{\partial \widehat{Y}(z, t)}{\partial a_{j}} \widehat{Y}^{-1}(z, t) d a_{j}
$$

в проколотой окрестности $\left\{z-a_{i}=0\right\}$. Следовательно, главная часть лорановского разложения $\omega_{2}$ совпадает с главной частью ее асимптотического 
разложения, т.е.

$$
\omega_{2}=\sum_{j=1}^{n}\left(\frac{\Phi_{M_{i}, j}^{i}(t)}{\left(z-a_{i}\right)^{M_{i}}}+\cdots+\frac{\Phi_{1, j}^{i}(t)}{z-a_{i}}+\varphi_{j}^{i}(z, t)\right) d a_{j},
$$

где $\left\{\Phi_{k, j}^{i}(t), \varphi_{j}^{i}(z, t)\right\}$ - голоморфные по $t$ матричные функции, $t \in D\left(t^{0}\right)$.

В случае, если $a_{i}$ - формально фуксовая особая точка, порядок полюса $M_{i}$ формы $\omega_{2}$ можно оценить.

Для каждого значения параметра $t$ запишем формальное фундаментальное решение в левелевском базисе (3.8)

$$
\widehat{Y}(z, t)=\widehat{U}(z, t)\left(z-a_{i}\right)^{\Lambda^{i}(t) / p}\left(z-a_{i}\right)^{\widehat{E}^{i}(t) / p} e^{Q^{i}(z, t)} .
$$

ЛЕмма 1. Если а $a_{i}$ - формально фуксовая иррегулярная особая точка системы (5.1), то матрица $\Lambda^{i}(t)$ в разложении (7.5) постоянна по $t$.

ДокАзАтельство. Пусть точка $a_{i}$ - неразветвленная формально фуксовая особая точка системы (5.1). Для фундаментального решения $Y(z, t) \sim \widehat{Y}(z, t)$ (формальное фундаментальное решение $\widehat{Y}(z, t)$ имеет вид (7.5)) должно быть выполнено равенство

$$
A_{-1}^{i}(t)=\lim _{z \rightarrow a_{i}}\left(\left(z-a_{i}\right) \frac{d Y(z, t)}{d z} Y^{-1}(z, t)-\frac{A_{-r_{i}-1}^{i}(t)}{\left(z-a_{i}\right)^{r_{i}}}-\cdots-\frac{A_{-2}^{i}(t)}{z-a_{i}}\right) .
$$

При построении левелевского базиса мы отмечали, что произведение

$$
\left(z-a_{i}\right)^{\Lambda^{i}(t)} \widehat{E}^{i}(t)\left(z-a_{i}\right)^{-\Lambda^{i}(t)}
$$

голоморфно, следовательно существует конечный предел

$$
\widetilde{E}^{i}(t)=\lim _{z \rightarrow a_{i}}\left(\left(z-a_{i}\right)^{\Lambda^{i}(t)} \widehat{E}^{i}(t)\left(z-a_{i}\right)^{-\Lambda^{i}(t)}\right) .
$$

И, далее, получаем

$$
A_{-1}^{i}(t)=P(t)\left(\Lambda^{i}(t)+\widetilde{E}^{i}(t)\right) P^{-1}(t),
$$

где матрица $P(t)$ определяется равенством $P(t)=U_{k}^{i}\left(a_{i}, t\right)$ для некоторого номера $k$ (матрица $U_{k}^{i}(z, t)$ в $\Omega_{k}\left(a_{i}\right)$ определяется своим асимптотическим разложением $\widehat{U}(z, t))$.

Таким образом, $\alpha_{k}^{i}=\rho_{k}^{i}+\lambda_{k}^{i}$, где $\alpha_{k}^{i}$ - собственные значения матрицы $A_{-1}^{i}(t)$. Собственные значения $\rho_{k}^{i}$ матрицы $\widehat{E}^{i}(t)$ постоянны, следовательно, каждое собственное значение $\alpha_{k}^{i}$ также не зависит от $t$, иначе оно изменялось бы сразу на целое число, что противоречит непрерывности матрицы $A_{-1}^{i}(t)$. Следовательно, матрица $\Lambda^{i}$ не зависит от $t$.

Пусть теперь точка $a_{i}$ - разветвленная формально фуксовая особая точка системы (5.1). Значит, формальное фундаментальное решение системы (5.1) в окрестности точки $a_{i}$ зависит от $\left(z-a_{i}\right)^{1 / p}, p \in \mathbb{N}$ и имеет вид (3.2). Сделаем замену независимой переменной $\xi=\left(z-a_{i}\right)^{1 / p}$ и перейдем к системе

$$
\begin{gathered}
\frac{d Y(\xi, t)}{d \xi}=\xi^{p-1} p A\left(\xi^{p}+a_{i}, t\right) Y(\xi, t) \\
\xi^{p-1} p A\left(\xi^{p}+a_{i}, t\right)=\frac{p A_{-r_{i}-1}^{i}(t)}{\xi^{p r_{i}+1}}+\cdots+\frac{p A_{-1}^{i}(t)}{\xi}+\cdots
\end{gathered}
$$


Аналогично случаю неразветвленной особой точки получаем, что выполнено равенство $\lambda_{k}^{i} / p+\rho_{k}^{i} / p=\alpha_{k}^{i}$ и элементы $\lambda_{k}^{i}$ матрицы $\Lambda^{i}$ не зависят от $t$.

ТеОРема 10. Пусть $a_{i}$ - формально фуксова особая точка и пусть $\widehat{Y}(z, t)-$ некоторое формальное голоморфное по $t$ фундаментальное решение системы (5.1) в окрестности точки $a_{i}$. Тогда для каждого значения $t \in D\left(t^{0}\right)$ найдется невырожденная матрица $C(t)$, коммутирующая $c Q^{i}(z, t)$, такая, что

$$
\widehat{Y}(z, t) C_{0} C(t)=\widehat{U}(z, t)\left(z-a_{i}\right)^{\Lambda^{i} / p}\left(z-a_{i}\right)^{\widehat{E}^{i}(t) / p} e^{Q^{i}(z, t)} .
$$

При этом, $\Lambda^{i}$ не зависит от $t$, матрицы $\Lambda^{i}, \widehat{E}^{i}(t)$ и $C(t)$ имеют одинаковый блочно-диагональный вид, матрицу $C(t)$ можно выбрать голоморфной по $t$ в некоторой окрестности $D^{\prime}\left(t^{0}\right) \subset D\left(t^{0}\right)$ и $C_{0}$ - постоянная невырожденная матрица.

Доказательство голоморфной зависимости $C(t)$ от $t$ аналогично доказательству теоремы 1 из [9; приложение 2].

СлЕДСТВиЕ. В окрестности формально фуксовой иррегулярной особой точки $a_{i}$ голоморфные по $t$ канонические решения $Y_{k}^{i}(z, t)$ системы (5.1), определенные по формальному решению $\widehat{Y}(z, t)$, представляются в виде

$$
Y_{k}^{i}(z, t) C_{0} C(t)=U_{k}(z, t)\left(z-a_{i}\right)^{\Lambda^{i} / p}\left(z-a_{i}\right)^{\widehat{E}^{i}(t) / p} e^{Q^{i}(z, t)}, \quad z \in \Omega_{k}\left(a_{i}\right) .
$$

ЛЕмма 2. Пусть $a_{i}$ - формалъно фуксова иррегулярная особая точка, тогда порядок полюса $M_{i}$ бормы $\omega_{2}$ вдоль гиперплоскости $\left\{z-a_{i}=0\right\}$ не превосходит максимума из минимального ранга Пуанкаре $r_{i}$ и иелочисленных разностей собственных значений матрицы $A_{-1}^{i}\left(t^{0}\right)$.

ДокАЗАтЕЛЬство. Рассмотрим систему канонических решений $Y_{k}^{i}(z, t)$, определенных по изомонодромному голоморфному фундаментальному решению $Y(z, t)$. Тогда с учетом равенства (7.9) каждое каноническое решение может быть представлено в виде

$$
Y_{k}^{i}(z, t)=U_{k}^{i}(z, t)\left(z-a_{i}\right)^{\Lambda^{i} / p} C^{-1}(t)\left(z-a_{i}\right)^{\mathcal{J}^{i} / p} e^{Q^{i}(z, t)} C_{0}^{-1} .
$$

При помощи равенства $d Y(z, t) Y^{-1}(z, t)=d Y_{k}^{i}(z, t)\left(Y_{k}^{i}(z, t)\right)^{-1}$ получаем асимптотическое разложение дифференциальной 1-формы $\omega_{2}$, определенной в (7.2), вдоль гиперплоскости $\left\{z-a_{i}=0\right\}$

$$
\begin{aligned}
\omega_{2} \sim \sum_{j=1}^{n} & \left(\frac{\partial \widehat{U}(z, t)}{\partial a_{j}} \widehat{U}^{-1}(z, t)+\widehat{U}(z, t) \frac{\partial Q^{i}(z, t)}{\partial a_{j}} \widehat{U}^{-1}(z, t)\right) d a_{j} \\
& +\sum_{j=1}^{n} \widehat{U}(z, t)\left(z-a_{i}\right)^{\Lambda^{i} / p} \frac{\partial C^{-1}(t)}{\partial a_{j}} C(t)\left(z-a_{i}\right)^{-\Lambda^{i} / p} \widehat{U}^{-1}(z, t) d a_{j} .
\end{aligned}
$$

Из соотношения (7.10) видно, что порядок $M_{i}$ полюса 1-формы $\omega_{2}$ вдоль гиперплоскости $\left\{z-a_{i}=0\right\}$ не превосходит минимального ранга Пуанкаре $r_{i}$ или максимальной разности формальных нормирований в точке $a_{i}$, т.е.

$$
M_{i} \leqslant \max _{k, j}\left\{\frac{\left|\lambda_{k}^{i}-\lambda_{j}^{i}\right|}{p}, r_{i}\right\},
$$

где $p$ - порядок ветвления, $\lambda_{j}^{i}$ - элементы матрицы $\Lambda^{i}$. 
Значит, с учетом равенства (7.6) получаем, что порядок полюса $M_{i}$ не превосходит минимального ранга Пуанкаре или максимума разностей собственных значений матрицы $A_{-1}^{i}\left(t^{0}\right)$, разность которых является целым числом.

ЗАмЕчАНИЕ 3. Форма изомонодромной деформации (5.1) вдоль гиперплоскости $\left\{z-a_{i}=0\right\}$, где точка $a_{i}-$ фуксова, описана в [10] и имеет вид

$$
\omega=\frac{A_{-1}^{i}(t)}{z-a_{i}} d\left(z-a_{i}\right)+\sum_{j=1}^{n} \sum_{k=1}^{M_{i}} \frac{\gamma_{j, k}^{i}(t)}{\left(z-a_{i}\right)^{k}} d a_{j}+\sum_{j=1}^{n} \psi_{j}^{i}(z, t) d a_{j},
$$

где матрицы $\psi_{j}(z, t)$ голоморфны, целое число $M_{i} \geqslant 0$ и равно максимальной целой разности собственных значений матрицы $A_{-1}^{i}\left(t^{0}\right)$ из $(2.4)$.

\section{§ 8. Глобальный вид формы деформации}

Теорема 11. Пусть $t=\left(a_{1}, \ldots, a_{n}\right)$. Дифференииалъная 1-форма $\omega$, определенная на $\mathbb{C P}^{1} \times D\left(t^{0}\right)$ и задающая изомонодромную деформацию (5.1), имеет вид

$$
\begin{aligned}
\omega=\sum_{i=1}^{n} & \sum_{k=1}^{r_{i}+1} \frac{A_{-k}^{i}(t)}{\left(z-a_{i}\right)^{k}} d\left(z-a_{i}\right) \\
& +\sum_{i=1}^{n} \sum_{j=1}^{n}\left(\frac{\Phi_{M_{i}, j}^{i}(t)}{\left(z-a_{i}\right)^{M_{i}}}+\cdots+\frac{\Phi_{1, j}^{i}(t)}{z-a_{i}}\right) d a_{j}+\sum_{j=1}^{n} \Psi_{j}(t) d a_{j},
\end{aligned}
$$

где матричные функиии $\left\{\Phi_{k, j}^{i}(t), \Psi_{j}(t)\right\}$ голоморфны в $D\left(t^{0}\right)$.

ДокАЗАТЕЛЬство. Вдоль каждой гиперплоскости $\left\{z-a_{i}=0\right\}$ справедливо разложение (7.3)-(7.4) или (7.11). Следовательно,

$\omega=\sum_{i=1}^{n} \sum_{j=1}^{n}\left(\frac{\Phi_{M_{i}, j}^{i}(t)}{\left(z-a_{i}\right)^{M_{i}}}+\cdots+\frac{\Phi_{1, j}^{i}(t)}{z-a_{i}}\right) d a_{j}+\sum_{i=1}^{n} \sum_{k=1}^{r_{i}+1} \frac{A_{-k}^{i}(t)}{\left(z-a_{i}\right)^{k}} d\left(z-a_{i}\right)+\Psi(z, t)$,

где $\Psi(z, t)$ - дифференциальная 1-форма, которая голоморфна на $\mathbb{C P}^{1} \times D\left(t^{0}\right)$ и, следовательно, не зависит от $z$. Таким образом, справедливо равенство (8.1).

\section{§ 9. Теорема о дифференциальной форме}

Приведенная ниже теорема хорошо известна для деформаций фуксовых систем (см. [9; лекция 13]). Также аналогичное утверждение (в несколько другой формулировке) доказано для допустимых деформаций систем с иррегулярными нерезонансными особыми точками [6]. Следующее доказательство в основном повторяет доказательство соответствующей теоремы из [9].

Теорема 12. Допустимая деформаиия (5.1) при $t=\left(a_{1}, \ldots, a_{n}\right)$ является изомонодромной тогда и только тогда, когда существует матричная дифференииальная мероморфная 1-форма $\omega$, определенная на $\mathbb{C P}^{1} \times D\left(t^{0}\right)$, с особенностями вдоль гиперплоскостей $\left\{z-a_{i}=0\right\}$ такая, что:

1) $\omega=A(z, t) d z$, при каждом фиксированном значении параметра $t \in D\left(t^{0}\right)$;

2) $d \omega=\omega \wedge \omega$. 
ДоказАТЕльство. Докажем необходимость. Пусть семейство (5.1) изомонодромно и $Y(z, t)$ - его изомонодромное голоморфное по обеим переменным фундаментальное решение. Рассмотрим дифференциальную 1-форму

$$
\omega=d Y(z, t) Y^{-1}(z, t)
$$

1-форма $\omega$ голоморфна на $\mathscr{M}=\mathbb{C P}^{1} \times D\left(t^{0}\right) \backslash \bigcup_{i=1}^{n}\left\{z-a_{i}=0\right\}$. Кроме того, она мероморфна в силу (8.1). Условие 1) выполнено по определению $\omega$. Условие 2) доказывается непосредственной проверкой (см. [9; лекция 13]).

Докажем достаточность. Рассмотрим систему дифференциальных уравнений

$$
d Y-\omega Y=0, \quad\langle(z, t), Y\rangle \in \mathscr{M} \times \mathbf{G L}(d, \mathbb{C}) .
$$

Она интегрируема с любыми начальными условиями $\left\langle\left(z^{0}, t^{0}\right), Y^{0}\right\rangle$. Действительно,

$$
d(d Y-\omega Y)=d^{2} Y-d(\omega Y)=\omega \wedge(d Y-\omega Y),
$$

т.е. выполнено условие Фробениуса (распределение, заданное дифференциальной 1-формой $\{d Y-\omega Y=0\}$, определенной на многообразии $\mathscr{M} \times \mathbf{G L}(d, \mathbb{C})$, интегрируемо) и система $d Y-\omega Y=0$ имеет локальное решение $Y(z, t)$, удовлетворяющее начальному условию $Y\left(z^{0}, t^{0}\right)=Y^{0}$. Фундаментальное решение $Y(z, t)$ аналитично по $t$.

Так как выполнено условие 1) из формулировки теоремы, следовательно, $Y(z, t)$ при фиксированном значении $t$ является фундаментальным решением соответствующей системы $d y / d z=A(z, t) y$ семейства. Матрицы монодромии, соответствующие фундаментальному решению $Y(z, t)$, не зависят от $t$.

Рассмотрим особую точку $a_{i}$. По теореме 4 фундаментальное решение $Y(z, t)$ имеет равномерное асимптотическое разложение $\widehat{Y}(z, t), z \in \Omega_{1}\left(a_{i}\right)$, коэффициенты которого голоморфно зависят от $t \in D\left(t^{0}\right)$.

Так как дифференциальная 1-форма $\omega$ является рациональной по $z$, то при каждом фиксированном $t \in D\left(t^{0}\right)$ существуют фундаментальные решения $Y_{1}^{i}(z, t), \ldots, Y_{N_{i}}^{i}(z, t)$ уравнения $d Y=\omega Y$, нормированные условием $Y_{k}^{i} \sim \widehat{Y}$ при $z \in \Omega_{k}\left(a_{i}\right)$ и голоморфные в секторах Стокса $\Omega_{k}\left(a_{i}\right)$.

Решения $Y_{k}^{i}(z, t)$ голоморфны и по $t \in D\left(t^{0}\right)$ (по теореме Коши-Ковалевской). Действительно, так как формальный ряд $\widehat{Y}(z, t)$ имеет голоморфные по $t$ коэффициенты, следовательно (согласно теореме 7 ), найдется голоморфная по $t$ функция, для которой $\widehat{Y}(z, t)$ является равномерным асимптотическим разложением. В силу условия 1$)$ решения $Y_{k}^{i}(z, t)$ являются решениями системы $d y / d z=A(z, t) y$ при фиксированном $t$.

Запишем систему (9.1) покоординатно, т.е.

$$
\frac{\partial Y}{\partial a_{j}}=\theta_{j} Y, \quad \omega=\sum_{j} \theta_{j} d a_{j}+\theta_{0} d z .
$$

Докажем теперь, что матрицы Стокса постоянны по $t$. Канонические решения $Y_{k}^{i}$ и $Y_{k+1}^{i}$ связаны соотношением $Y_{k+1}^{i}(z, t)=Y_{k}^{i}(z, t) S_{k}^{i}(t)$, где $S_{k}^{i}(t)$ - матрица 
Стокса. Тогда получаем

$$
\begin{aligned}
\theta_{h} Y_{k+1}^{i} & =\frac{\partial Y_{k+1}^{i}}{\partial a_{h}}=\frac{\partial\left(Y_{k}^{i} S_{k}^{i}\right)}{\partial a_{h}}=\frac{\partial Y_{k}^{i}}{\partial a_{h}} S_{k}^{i}+Y_{k}^{i} \frac{\partial S_{k}^{i}}{\partial a_{h}} \\
& =\theta_{h} Y_{k}^{i} S_{k}^{i}+Y_{k}^{i} \frac{\partial S_{k}^{i}}{\partial a_{h}}=\theta_{h} Y_{k+1}^{i}+Y_{k}^{i} \frac{\partial S_{k}^{i}}{\partial a_{h}}
\end{aligned}
$$

Следовательно, $\partial S_{k}^{i}(t) / \partial a_{h}=0$, так как $Y_{k}^{i}(z, t)$ - невырожденная матрица.

Таким образом, матрицы Стокса не зависят от параметров деформации. Следовательно, в силу равенства (4.6), матрицы формальной монодромии $\widehat{G}^{i}$ также постоянны по $t$.

\section{$\S 10$. Расширение набора параметров}

Предыдущие утверждения остаются справедливы и в том случае, когда деформируются положения особых точек $a_{i}$, и для каждой неразветвленной особой точки деформируются коэффициенты $\beta_{h}$ полиномов, которые являются элементами матриц $Q^{i}(z)$, за исключением коэффициентов при старших степенях $\left(z-a_{i}\right)^{-1}$. То есть в качестве набора параметров служит $t=\left(a_{1}, \ldots, a_{n}\right.$, $\left.\beta_{1}, \ldots, \beta_{H}\right)$. В этом случае общий вид формы деформации станет следующим:

$$
\begin{aligned}
\omega=\sum_{i=1}^{n} & \sum_{k=1}^{r_{i}+1} \frac{A_{-k}^{i}(t)}{\left(z-a_{i}\right)^{k}} d\left(z-a_{i}\right) \\
& +\sum_{i=1}^{n} \sum_{j=1}^{n}\left(\frac{\Phi_{M_{i}, j}^{i}(t)}{\left(z-a_{i}\right)^{M_{i}}}+\cdots+\frac{\Phi_{1, j}^{i}(t)}{z-a_{i}}\right) d a_{j}+\sum_{j=1}^{n} \Psi_{j}(t) d a_{j} \\
& +\sum_{i=1}^{n} \sum_{h=1}^{H}\left(\frac{\bar{\Phi}_{N_{i}, h}^{i}(t)}{\left(z-a_{i}\right)^{N_{i}}}+\cdots+\frac{\bar{\Phi}_{1, h}^{i}(t)}{z-a_{i}}\right) d \beta_{h}+\sum_{h=1}^{H} \bar{\Psi}_{h}(t) d \beta_{h}
\end{aligned}
$$

где матричные функции $\left\{\Phi_{k, j}^{i}(t), \Psi_{j}(t) \bar{\Phi}_{k, h}^{i}(t), \bar{\Psi}_{h}(t)\right\}$ голоморфны в $D\left(t^{0}\right)$.

\section{Список литературы}

[1] Б. Риман, Сочинения, ОГИЗ, М., 1949; пер. с нем.: В. Riemann, Gesammelte mathematische Werke und wissenschaftlicher Nachlass, Teubner, Leipzig, 1892.

[2] M. Jimbo, T. Miwa, K. Ueno, "Monodromy preserving deformation of linear ordinary differential equations with rational coefficients. I. General theory and $\tau$-function", Phys. D, 2:2 (1981), 306-352.

[3] A. A. Bolibruch, S. Malek, C. Mitschi, "On the generalized Riemann-Hilbert problem with irregular singularities", Expo. Math., 24:3 (2006), 235-272.

[4] W. Balser, W. B. Jurkat, D. A. Lutz, "A general theory of invariants for meromorphic differential equations. II. Proper invariants", Funkcial. Ekvac., 22:3 (1979), 257-283.

[5] Y. Sibuya, "Stokes phenomena", Bull. Amer. Math. Soc., 83:5 (1977), 1075-1077.

[6] А.Р. Итс, А. А. Капаев, В. Ю. Новокшенов, А. С. Фокас, Трансценденты Пенлеве. Метод задачи Римана, Ин-т компьютерных исследований, М.-Ижевск, 2005; пер. с англ.: А. S. Fokas, A.R. Its, A. A. Kapaev, V.Yu. Novokshenov, Painlevé transcendents. The Riemann-Hilbert approach, Amer. Math. Soc., 128, Princeton Univ. Press, Providence, RI, 2006. 
[7] М. В. Федорюк, "Изомонодромные деформации уравнений с иррегулярными особенностями", Матем. сб., 181:12 (1990), 1623-1639; англ. пер.: M. V. Fedoryuk, "Isomonodromy deformations of equations with irregular singularities", Math. USSR-Sb., 71:2 (1992), 463-479.

[8] I. M. Krichever, "Isomonodromy equations on algebraic curves, canonical transformations and Whitham equations", Mosc. Math. J., 2:4 (2002), 717-752.

[9] А. А. Болибрух, Обратнъе задачи монодромии в аналитической теории дифберенциальных уравнений, МЦНМО, М., 2009.

[10] А.А. Болибрух, "Дифференциальные уравнения с мероморфными коэффициентами", Совр. пробл. матем., 1, МИАН, М., 2003, 29-82; англ. пер.: A. A. Bolibrukh, "Differential equations with meromorphic coefficients", Proc. Steklov Inst. Math., 272, suppl. 2 (2011), 13-43.

[11] А.А. Болибрух, "Об изомонодромных слияниях фуксовых особенностей”, Локалъные и глобалъные задачи теории особенностей, Тр. МИАН, 221, Наука, M., 1998, 127-142; англ. пер.: А. A. Bolibrukh, "On isomonodromic confluences of Fuchsian singularities", Proc. Steklov Inst. Math., 221 (1998), 117-132.

[12] А.А. Болибрух, "Регулярные особые точки как изомонодромные слияния фуксовых", УМН, 56:4 (2001), 135-136; англ. пер.: A. A. Bolibrukh, "Regular singular points as isomonodromic confluences of Fuchsian singular points", Russian Math. Surveys, 56:4 (2001), 745-746.

[13] D. V. Anosov, "Concerning the definition of isomonodromic deformation of Fuchsian systems", Ulmer Seminaire Euber Funktionalysis und Differentialgleichungen, 2 (1997), 1-12.

[14] A. Bolibruch, "Inverse problems for linear differential equations with meromorphic coefficients", Isomonodromic deformations and applications in physics (Montreal, QC, Canada, 2000), CRM Proc. Lecture Notes, 31, Amer. Math. Soc., Providence, RI, 2002, 3-25.

[15] В. Вазов, Ассимптотические разложения решений обыкновенных дифференциалъных уравнений, Мир, М., 1968; пер. с англ.: W. Wasow, Asymptotic expansions for ordinary differential equations, Wiley, New York-London-Sydney, 1965.

[16] W. Balser, W. B. Jurkat, D. A. Lutz, "A general theory of invariants for meromorphic differential equations. I. Formal invariants", Funkcial. Ekvac., 22:2 (1979), 197-221.

[17] Р. Р. Гонцов, "Уточненные неравенства Фукса для систем линейных дифференциальных уравнений", Изв. РАН. Сер. матем., 68:2 (2004), 39-52; англ. пер.: R. R. Gontsov, "Refined Fuchs inequalities for systems of linear differential equations", Izv. Math., 68:2 (2004), 259-272.

[18] R. Schäfke, "Formal fundamental solutions of irregular singular differential equations depending upon parameters", J. Dynam. Control Systems, 7:4 (2001), 501-533.

[19] B. Malgrange, "Deformations of differential systems. II", J. Ramanujan Math. Soc., 1:1-2 (1986), 3-15.

[20] V. Heu, "Universal isomonodromic deformations of meromorphic rank 2 connections on curves", Ann. Inst. Fourier (Grenoble), 60:2 (2010), 515-549.

[21] M. Bertola, M. Y. Mo, "Isomonodromic deformation of resonant rational connections", IMRP Int. Math. Res. Pap., 2005, № 11, 565-635.

Ю. П. Бибило (Yu. P. Bibilo)

Национальный исследовательский университет

"Высшая школа экономики", г. Москва

E-mail: y.bibilo@gmail.com
Поступила в редакцию 16.03.2011 